\title{
The Concept of Authoritative Parenting and It's Effects on Academic Achievement
}

\begin{abstract}
It has been recommended that parenting styles play a meaningful part in the growth of children and their perspectives. Authoritative parents may be considered the most attractive of the sorts, due to their distinguished symmetry between structure and affection. Child development professionals generally categorize the authoritative parenting style as the greatest methodology to parenting. This research study entails a total of 290 participants in survey conducted, regarding parenting and academic achievement. It was hypothesized that students with high authoritative parents would have higher learning focused goals and earns a higher college GPA than students with low authoritative parents. It was also theorized that students with high authoritative parents would place a greater amount of blame of academic failures on lack of effort than those with low authoritative parents. Following the completion of this survey and widespread analysis, there has been an indication that authoritative parenting is reasonably related to the manner in which a student contemplates and performs in his or her studies.
\end{abstract}

Research Article

Volume 3 Issue 6 - 2015

Triana Rego*

Fairleigh Dickinson University, USA

*Corresponding author: Triana Rego, Fairleigh Dickinson University, USA, Email: tregoventoso@gmail.com

Received: July 22, 2015 | Published: October 14, 2015

\section{Introduction}

\section{The concept of authoritative parenting and its effects on academic achievement}

Parenting has been suggested to take a significant role in the development of children and their viewpoints. During the 1960s, Diana Baumrind, a developmental psychologist, explained three different kinds of parenting styles, based on her research with preschoolers [1]. This researcher would currently like to discuss one of the main kinds of parenting styles, authoritative parenting. Authoritative parents may be the most appealing of the types because of their well-known balance between structure and affection. They listen to their children, allow them to express their feelings, and inspire them to talk about options. Huver et al. [2] suggested that two features of personality-mirroring social relations, extroversion and agreeableness, are associated with supportiveness, while emotional solidity is related to sensible firmness. Authoritative parents encourage independence, manage unbiased and coherent discipline, and display warmth. Their parenting techniques allow them to place boundaries, and demonstrate consequences and expectations on their children's conduct. Parents should be educated about authoritative parenting and the effects it can have on their children's academic achievement.

Child development experts typically classify the authoritative parenting style as the best-known methodology to child rearing. Unlike formerly promoted styles regarding low sensitivity and high demandingness, known as authoritarian parenting, or high sensitivity and low demandingness, identified as permissive parenting, authoritative parenting implicates high levels of both responsiveness and demandingness; the outcome is a suitable assortment of heartfelt support and consistent firmness [3]. Children raised by authoritative parents tend to be more proficient, cheerful, and successful [1]. Children of authoritative parents are likely to believe that they have the ability to conquer new problems and complete new tasks. These confident people are also most likely to understand that disappointments may occur when they do not try hard enough. They blame their failures on their lack of effort, rather than any lack of intelligence. Numerous intensive analyses and interviews have offered the idea that, globally, young people display a greater appreciation of success, after engaging in "hard work" [4].

It is clear that when a person has this kind of self-assurance, it travels along with the urge to learn and do more. Learning focused goals involve the objectives of people whose main priorities are to learn from their schoolwork and experiences, rather than succeed merely to demonstrate a point to another individual. When a person has learning focused goals, he or she is most likely to become involved in the focused activities, ultimately learning more and earning higher grade point averages. A caretaker's aptitude to express personal interest in learning is vital in gaining and stimulating his or her children's enthusiasm and inquisitiveness in their regulation and life experiences [5]. As the students uncover and build their own understanding, this "love of learning" may be therapeutic and inspiring. These are the variables in which this researcher would like to examine.

In order to conduct this analysis, past research has been utilized as an introductory point. The first study brought into play was commenced in order to determine the impact of different parenting styles on first-year students' adaptation to college. This study was steered by asking 80 first-year students from two-parent families to complete the Parental Authority Questionnaire and the Tests of Reactions and Adaptations to College, during their second week of college [6]. It was discovered that the students with authoritative parents had more optimistic characters, underwent less anxiety, were more willing to ask peers and professors for help, and were more prepared for exams than students with other types of parents. The behavioral and belief differences between permissive and authoritarian parenting were suggested to be insignificant. Fathers' parenting methods were suggested to have a greater effect on the students than the parenting methods of the mothers. 
The information presented can be rather valuable, especially since it explains the mistakes in so many earlier studies, which suggested that a mother's love had a greater effect than a father's love, on child development. Young, Miller, Norton, and Hill (1995) found that apparent paternal love and caring was just as foretelling, of children's life gratifications and sense of well being, as maternal love and caring, if not more [7]. Studies regarding fathers are even starting to explain substance abuse, psychopathology, depression, behavior issues, and borderline personality disorder [8]. The results of this past research take great emphasis on the idea that students with authoritative parents are more likely to make their college studies a top priority, while attaining less stress and negativity, than students with authoritarian and permissive parents. With a positive outlook on life, students are well aware that they can achieve great success with the right amount of effort.

Further exploration took place, in order to discover a bond between authoritative parenting and the blame of academic failures on lack of effort. The purpose of this article, Academic Achievement in an Age of Irresponsibility, is to support the notion that the failure of parents to allow their children to take responsibility for their actions, ultimately, points the children into very irresponsible directions. These children may unfortunately continue to blame others for their mistakes, ultimately causing them to continue to make poor choices as adults. Moreover, modifications in particular parenting types are related to shifts in juvenile delinquency, most notably the changes categorized by a decline in responsiveness or an rise or drop in demandingness; This adequately describes authoritarian parents [9]. Overly permissive parents, who "baby" their children, rather than providing them with clear and consistent guidelines, may eventually lead them to a very unsuccessful future.

The aforementioned article discusses the perception that contemporary American culture has begun to protect children from the consequences of their own actions by raising them to be responsible for their own actions. Rather than helping students with their schoolwork, more parents and teachers are providing the kids with independence and being more authoritative. This act is becoming known as "good parenting" [10]. The study suggests instructors begin to reflect on the new tendency for students to show carelessness and very little determination in the face of academic difficulty. Parents and teachers are recommended to permit their children and students to do things on their own and fail at times; if not, they may continue depend on others and never excel.

A beneficial approach to success is to focus on goals that involve your own learning, rather than demonstrating your worth to others. After some research, an article on undergraduate students' goal orientations and their relationship to perceive parenting styles supported the information in this research article, regarding the connection between learning focused goals and authoritative parenting. The purpose of this study was to observe the connections among the three parenting styles and the mastery and functioning goal orientations of undergraduate college students. Parent educational accomplishment and parent involvement were also included in the analysis for the purposes of controlling the effects of these variables on student goal direction. The study involved of 311 undergraduate students, who were currently taking teaching or psychology courses.
The first goal has been labeled a mastery goal, learning goal, or task involved. Students who are concerned with mastery goals enjoy learning new abilities and cultivating their knowledge and proficiency. The second is a functioning goal or personality involved. Students with performance goals are more apprehensive about demonstrating their ability or avoiding negative rulings of their capability. Students with authoritative parents were found to be more likely to have mastery goal behaviors, such as independence, the inclination to try new things, the ability to enjoy school and work, and intrinsic motivation, than students with other kinds of parents [11].

If authoritative parenting is related to a higher GPA in college, then a student with high authoritative parents will be most likely to earn a higher GPA in college than those with low authoritative parents. If authoritative parenting is associated to learning focused goals, then a student with high authoritative parents will be most likely to have greater learning focused goals than those with low authoritative parents. If authoritative parenting is related to the amount of blame of academic failures a student places on lack of effort, then a student with high authoritative parents will be most likely to place a higher amount of blame of academic failures on lack of effort.

\section{Method}

\section{Participants}

There were a total of 290 participants in the survey conducted, regarding parenting and academic achievement. Of the participants, 257 were females, a total of $88.6 \%$, while 33 of the participants were males, equaling $11.4 \%$. The participants varied from 18 to 37 years of age. The average age of the participants who partook in this survey was 20.35 . A small $1.4 \%$ of the group was solely Arab, and $9.3 \%$ were solely Asian/Pacific Islander. Of the participants, $17.6 \%$ were only Black/African American, and the majority of them were just Hispanic or Latino, at a great $29.0 \%$. $27.6 \%$ of them were White/Caucasian, and $4.8 \%$ of the group identified themselves as an unlisted race. Of the group, $8.7 \%$ of the participants identified themselves as multiracial, or coming from more than one race.

\section{Measures}

The survey at hand is made up of multiple-choice questions. A total of 38, out of the 290 participants, failed to answer questions relating to college GPA. A total of 2 students failed to answer questions related to learning focused goals, while a total of 2 students failed to answer questions related to the blame of failure on lack of effort. Some of the questions provide very specific yes or no answers or direct responses, such as races or precise numbers; others may consist of a range of numbers or diverse responses.

The distinct responses included in Sections 2, 3, and 4, which hold the questions about parenting, are as follows: "Not true at all", "slightly true", "moderately true", or "extremely true". Other responses in these sections include: "Very like", "moderately like", "moderately unlike", "very unlike", or "check this box, if you do not want to provide an answer to this question". The remainder of these retorts include: "Strongly disagree", "disagree", "sort of disagree", "neither agree nor disagree", "sort of agree", "agree", and "strongly agree". An example of one of the statements, regard- 
ing type of parenting, is "My mother appeared to understand my problems and worries." The answers ranged on a 1 to 4 scale, with "very like" as the first point and "very unlike" as the fourth point. A question in Section 1 reads, "What is your current overall college GPA?". The answers were arranged on a 1 to 7 scale with "less than 1.5 " as the first point and "4.0" as the seventh point.

In Section 5, questions 1 through 4 assess the learning focused goals of the participants, providing them with statements, such as "I prefer challenging and difficult classes, so that I'll learn a great deal." The answers ranged on a 1 to 7 point scale, with "strongly agree" as the first point and "strongly disagree" as the seventh point. In Section 5, questions 16 and 17 assess the blame of academic failures on lack of effort, providing them with answers that range on a 1 to 7 point scale, with "strongly disagree" as the first point and "strongly agree" as the seventh point. The two statements read: "I can perform any school task, if someone tells me the right way to do it" and "Failing a task at school is usually caused by applying an incorrect strategy."

\section{Procedure}

The study involved in this research included 89 multiple-choice questions, within six sections on an online survey. The survey was available on a private university's Sona system, offered to students in Psychology courses, who may have been interested in participating. A participant would not be able to partake in the survey without Internet access. The minimum duration that a student took to focus on the effect of parenting and academic achievement was 8 minutes, while the maximum was 58 minutes. The mean duration of the study was 14.34 minutes.

\section{Results}

An independent samples $t$-test was conducted to compare the college GPA of students that have high authoritative parents and students that have low authoritative parents. It was hypothesized that students with high authoritative parents would earn a higher college GPA than students with low authoritative parents. There was an adequate difference in the college GPA of students based on the authoritativeness of their parents $(t(260)=-2.337, p<.05)$. The results indicate that there are distinctions in students' college GPAs based on the authoritativeness of their parents. Specifically, students with high authoritative parents earned moderately higher college GPAs $(\mathrm{M}=5.11, \mathrm{SD}=0.773)$ than students with low authoritative parents $(\mathrm{M}=4.89, \mathrm{SD}=0.796)$.

An independent samples $t$-test was conducted to compare the learning focused goals of students that have high authoritative parents and students that have low authoritative parents. It was hypothesized that students with high authoritative parents would have greater learning focused goals than students with low authoritative parents. There was a substantial difference in the learning focused goals of students based on the authoritativeness of their parents $(t(286)=-2.796, p<.05)$. The results indicate that there are differences in learning focused goals based on the authoritativeness of their parents. Unambiguously, the students with high authoritative parents had greater learning focused goals $(\mathrm{M}=23.77, \mathrm{SD}=5.708)$ than the students with low authoritative parents $(\mathrm{M}=21.93, \mathrm{SD}=5.466)$.

An independent samples $t$-test was conducted to compare the amount of blame of academic failures, placed on lack of effort, by students that have high authoritative parents and students that have low authoritative parents. It was hypothesized that students with high authoritative parents would place a greater amount of blame of academic failures on lack of effort than students with low authoritative parents. There was a substantial difference in the amount of blame of academic failures placed on lack of effort by students, based on the authoritativeness of their parents ( $t$ $(286)=-2.142, p<.05)$. The results indicate that there are differences in the amount of blame of academic failures placed on lack of effort by students, based on the authoritativeness of their parents. In detail, the students with high authoritative parents placed a greater amount of blame of academic failures on lack of effort ( $M=11.00, D F=1.537)$ than the students with low authoritative parents $(\mathrm{M}=10.54, \mathrm{DF}=2.018)$ (Table 1$)$.

Table 1: Descriptive Statistics for Authoritative Parenting and its Effects on Academic Achievement.

\begin{tabular}{|c|c|c|c|c|c|c|c|}
\hline \multicolumn{5}{|c|}{ Low Authoritativ } & \multicolumn{3}{|c|}{ High Authoritative } \\
\hline Factor & $n$ & $M$ & $(S D)$ & $n$ & $M$ & $(S D)$ & $t p$ \\
\hline College GPA & 1484.89 & $(0.796)$ & 114 & 5.11 & $(0.773)$ & -2.337 & 0.020 \\
\hline Learning Focused Goals 160 & 21.93 & $(5.466)$ & 128 & 23.77 & $(5.078)$ & -2.796 & 0.006 \\
\hline $\begin{array}{l}\text { Blame of Academic Failure } \\
\qquad 160\end{array}$ & 10.54 & $(2.018)$ & 128 & 11.00 & $(1.537)$ & -2.142 & 0.033 \\
\hline
\end{tabular}

\section{Discussion}

Following the completion of this survey and widespread analysis, there has been an indication that authoritative parenting is moderately related to the manner in which a student contemplates and performs in his or her studies. It has been suggested that, if a child grows up with an authoritative parent, he or she will most likely blame failure on his or her own shortage of effort, rather than lack of intelligence. The student's goals will most likely be based on learning, rather than demonstrating his or her own intelligence or worth to another person. Authoritative parenting may affect a student's scholarly mindset, as well as his or her grade point average in college. It has been theorized that parenting styles control the bonds among parenting exercises, achievement, and conduct; Furthermore, behavioral control amplified the rapport between home-based involvement and suitable school performance [12].

In regards to the first literary review, presented earlier in this research article, the impact of different parenting styles, concerning first-year students' adaptation to college, supports the relationship between college GPA and authoritative parenting, analyzed in this study. This study suggests that students with high authoritative parents have a moderately greater likelihood 
to attain high college GPAs than students with other types of parents. Past research proposed that different parenting styles have helped to extend the growth of students' personalities and nature [6]. It also provided the support that these individual characteristics, eventually, influence and may be represented through the students' preliminary adjustment to college. After an time-consuming study, [13] Hoover discusses students' reactions to parental involvement on campus by suggesting that they essentially view this immersion as "more of a help than a headache". Certain representatives suppose schools should involve parents, predominantly those who did not attend universities themselves, on a more extensive basis. The latest National Survey of Student Engagement indicates engaged parents may help their children achieve [13]. Ultimately, students with high authoritative and plugged-in, parents have been suggested to hold a greater likelihood of optimistic attitudes and well adjustment to college, possibly leading them to higher GPAs. This information has been supported through this examination, due to the reasonable connection between high authoritative parents and the high college GPAs of students.

On the topic of academic achievement, past research of this accomplishment in an age of irresponsibility supports the information provided in this research paper, regarding the connection between authoritative parenting and the blame of academic failures on lack of effort. Students with high authoritative parents are more likely to blame their own lack of effort on their failures, rather than other people or causes. This is because authoritative parents have a beneficial balance between strictness and nurturing. They give their children guidance, but also give them the independence to learn and work on their own. Past research, which educates us on the matter of the "stress of "No Child Left Behind", cannot be understood, as parents or teachers pick a child up and carry him or her, every time he or she falls [10]. Authoritative parents serve as role models, by providing steady rules and nurturing their children, while also letting their 'baby birds' fly on their own. The findings insist that children, who are constantly dependent on their caretakers and get everything done for them, without any consequences for their actions, tend to blame others or their own intelligence for their failures. They do not have any sense of responsibility or confidence, which may, unfortunately, cause them to difficulty when attempting to stand their ground or function as an autonomous person.

The outcomes of confidence and autonomy can help a student to form beneficial goals in life. In regards to the third literary review previously presented in this research paper, the impact of undergraduate students' goal focuses and their relationship to apparent parenting styles supports the relationship between authoritative parenting and learning focused goals, analyzed in this study. The study highlighted in this research article suggests that students with high authoritative parents are more likely to have learning focused than students with other types of parents. Past research proposed that the relationship between a mother's authoritativeness and mastery orientation has a greater impact on girls than it does boys [11]. This information displays a great connection to the material provided in this research article, due to fact that the significance of this analysis is based on a majority of female participants. Past research also suggests that a mother's authoritativeness in the home may also be more prominent in the expansion of the self-esteem of females, rather than boys. Mater- nal comfort has been implied to cause a more significant rise in the connection between maternal school-based involvement and children's achievement in girls, rather than boys [12].

As implied initially, the effects of the manner in which an individual decides to parent his or her children may help to form a child's outlook on life, primarily when it comes to education and their academic achievement. Parents should be educated on the findings of this study, so that they may learn to find a "happy medium", when it comes to their nurturing methods. They may learn to provide disciplinary rules for their children, while also showing them the love and support necessary, to assist in the development of their self-images. The information provided in this research article can be used in order to educate all foster and adoptive parents, before they begin their duties as surrogate caretakers. Of the approximately 500,000 children in the U.S. who are presently being aided by the foster care system, infants and toddlers make up the largest part; Although these darlings, who are inclined to spend the longest time in the system, are at the utmost risk for physical and mental well being, as well as developmental concerns, slight research has been steered to discover their experiences and developmental aftermaths [14]. Puig [14] described a study in which "birth relatives and foster relatives of several generations integrate Early Intervention amenities to help a toddler living within the foster care system". The foster system could unquestionably benefit from this knowledge and positively even provide a better name for its self. It is in my belief that the information also would be highly useful for parents, who choose to learn how the treatment inflicted upon their children can affect them indefinitely.

There were some limitations in the analysis involving the particular topic at hand, diversity of students, and location of the study. When it comes to discussing family and personal matters, many individuals seem to feel uncomfortable. Although some students may have indicated that their parents are authoritative, this information could be fabricated and untruthful. Another limitation was the lack of variety amongst the genders of the participants. Out of 290 participants, merely 33 of them were males, ultimately causing this study's results to exclusively be truthful about females. Additional limitations, involving the location and diversity of the study, include the fact that the survey was only available to participants online, on Fairleigh Dickinson University's Sona Systems website; therefore, the participants needed to have Internet access. In order to take this survey, a student was required to be taking a Psychology course at Fairleigh Dickinson University, ultimately not leaving much room for variety in this analysis.

With the scrutinized information specified in this research paper, individuals could make an effort to find out more on the topic of authoritative parenting related to college GPA, learning focused goals, and effort failure. A beneficial way to further analyze this research would be to conduct a study, involving adults who have already completed their education. Does past authoritative parenting ultimately affect an adult's future in his or her career or relationships? Do these students grow up to parent similarly to the way they were raised? For instance, researchers have frequently contemplated the widely accepted belief that abused children are likely to become abusive parents [15]. This information could be further questioned and possibly supported by the inquiry of the 
parenting methods employed on these children and their future adult-selves.

Additional future questions that would be suitable to be answered would be whether or not the complicated factors, such as the strictly online examination and lack of male participants, affected the outcome of the study presented in this research article. Research conducted by Stright \& Yeo [12] suggested that the link between warmth and achievement was clearer for girls than boys, while the link between home-based school involvement and achievement was stronger for boys than for girls. If the outcome is positively correlated, the information can be used to inform parents about the affiliation between their parenting techniques and their child's overall future. School-focused parenting exercises may predict success and behavior after amending parenting styles [12].

It has come to my attention that the information in this study, relating authoritative parenting to the academic achievements of children, may be rather expedient to parents, who wish to increase their child's probability of having a successful future. Since authoritative parents act as role models and display the same conducts that they anticipate from their children, the children are more likely to adopt these behaviors [1]. Castro et al. [16] conducted research which suggested that, collectively, parents' principles, conducts, and talents account for $37 \%$ of the adjustment in the emotion perception skills of children, even following the control of parent and child expressive precision. Their conclusions offer the significance of the family environment in the expansion of children's emotion perception ability in middle childhood and support accruing indications advocating imperative age-related changes in the link between parental emotion socialization and child emotional expansion [16]. As these parents display good emotional consideration and management, their children also learn to control their own sentiments and learn to understand those of others, as well. Authoritative parents provide their children with the consent to act individually, teaching the children that they are efficient enough to complete tasks on their own; this aids them in the adoption of high self-esteem and confidence.

Blaming academic failures on lack of effort infers that the student's self-esteem is most likely higher than a student who blames academic failures on lack intelligence. High self-esteem may offer an individual a positive mentality and the will to never give up. "Over more than a generation, the awareness that children need nurturance of a high self-esteem, in order to be developmentally vigorous, has had expansive recognition in Western psychology; The emphasis on self-esteem is an extension to the theory that comprehensive self-esteem is virtually tantamount to mental health [17]" It is in this researcher's belief that a high college GPA reasonably suggests that a student has attained much knowledge in the subject of his or her choice and has had the confidence to utilize that knowledge. Much like learning focused goals, this can provide a student with a greater advantage in the job industry, which can ultimately decide the success of his or her career and future. Wouldn't parents and caretakers find it advantageous if they could determine their children's imminent success, simply by following the recommendations of authoritative parenting?.

\section{References}

1. Cherry K (2014) What is authoritative parenting?. About Psychology.

2. Huver R, Otten R, De Vries H, Engels R (2010) Personality and Parenting Style in Parents of Adolescents. J Adolesc 33(3): 395-402.

3. Larzelere R (2013) Authoritative Parenting: Synthesizing Nurturance and Discipline for Optimal Child Development In: Sheffield Morris A \& Harrist A (Eds.), American Psychological Association, Washington D.C., USA, pp. 1-280.

4. Mendick H, Allen K, Harvey L (2015) “We Can Get Everything We Want if We Try Hard": Young People, Celebrity, Hard Work. British Journal of Educational Studies 63(2): 161-178.

5. Penman J, Ellis B (2009) Regional Academics' Perceptions of the Love of Learning and Its Importance for Their Students. Australian Journal of Adult Learning 49(1): 148-168.

6. Smith GJ (2006) The impact of different parenting styles on first-year college students' adaptation to college. Biennial Conference of the Society for Research in Human Development 1-4.

7. Rohner R (1998) Father Love and Child Development: History and Current Evidence. Psychological Science 157-161.

8. Bower B (1992) Same Family, Different Lives. Science New 376-378.

9. Schroeder R, Mowen T (2014) Parenting Style Transitions and Delinquency. Youth \& Society 46(2): 228-254.

10. Dichele AM (2006) Academic achievement in an age of irresponsibility. The Journal of Culture and Education 10(1) 29-33.

11. Gonzalez A, Greenwood G, Wenhsu J (2001) Undergraduate students. College Student Journal 35(2): 1-8.

12. Stright A, Yeo K (2014) Maternal Parenting Styles, School Involvement, and Children's School Achievement and Conduct in Singapore. Journal of Educational Psychology 106(1): 301-314.

13. Hoover E (2008) Surveys of Students Challenge "Helicopter Parent" Stereotypes. Chronicle of Higher Education 54(21): A22-A22.

14. Puig V (2013) Re-Imagining Language, Culture, and Family in Foster Care. Early Child Development and Care 183(11): 1625-1645.

15. Kaufman J, Zigler E (1987) Do abused children become abusive parents? American Journal of Orthopsychiatry 57(2): 186-192.

16. Castro V, Halberstadt A, Lozada F, Craig A (2015) Parents' EmotionRelated Beliefs, Behaviours, and Skills Predict Children's Recognition of Emotion. Infant and Child Development 24(1): 1-22.

17. Persinger J (2012) An Alternative to Self-Esteem: Fostering SelfCompassion in Youth. Communique 40(5): p20. 
